\title{
Spatial development of multiple-gap states in nonequilibrium superconductors
}

\author{
H. Schreyer, W. Dietsche, and H. Kinder \\ Physik Department der Technischen Universität München, D-8046 Garching, West Germany \\ P. Leiderer \\ Fachbereich Physik der Universität Mainz, D-6500 Mainz, West Germany \\ (Received 21 September 1984)
}

\begin{abstract}
We have studied the gap instability in a superconductor under tunneling injection at high voltages by probing the spatial distribution of the phonon emission. A high sensitivity was achieved by using the fountain pressure of superfluid helium for detecting the phonons. Spatial structures were observed at gap depressions as small as $2 \%$. From their spatial development we find that the quasiparticles diffuse into regions where their density is higher.
\end{abstract}

Nonequilibrium superconductivity is attracting increased interest from both the fundamental ${ }^{1}$ and the technological $^{2}$ points of view. The nonequilibrium states are most often produced by tunnel injection of quasiparticles. $^{3-6}$ A particularly striking feature is the occurrence of two (or more) distinct energy gaps implying the coexistence of several superconducting phases. Dynes, Narayanamurti, and Garno ${ }^{4}$ found two different gaps when they injected quasiparticles at just the gap-sum voltage. The same phenomenon was investigated by Gray and Willemsen, ${ }^{5}$ and was explained by negative-resistance switching near the steep rise at the gap sum in the $I-V$ characteristic. However, this model was not applicable to the case of injection of quasiparticles at high voltages, far above the gap sum, where Iguchi and Langenberg ${ }^{6}$ still found multiple gaps. The latter phenomenon is not well understood because at high-voltage injection the tunneling current is almost independent of the energy gap. Thus a gap instability can not be sustained by the injection current alone.

Therefore, phenomenological models ${ }^{7,8}$ were proposed which required lateral quasiparticle diffusion. In' these models the chemical potential $\mu^{*}$ decreases with increasing quasiparticle density under certain unstable conditions. This leads to a diffusion against the density gradient ("diffusive instability"). However, these concepts were not supported ${ }^{9}$ by more rigorous treatments ${ }^{10}$ which yielded a positive diffusion coefficient throughout, and an instability occurring only through the coherence factor ("coherent instability"). On the other hand, there was a possibility that the multiple gaps had been caused by inhomogeneous tunneling injection, i.e., by irregularities in the tunnel barrier particularly in view of the fact that Gray and Willemsen ${ }^{5}$ did not observe the phenomenon at all.

To gain more insight, spatially resolved techniques were developed more recently. Akoh and Kajimura ${ }^{11}$ probed the gap by a microjunction array. Kotani, Suzuki, and Iguchi $^{12}$ measured the local phonon-emission rate via the heat emitted into the liquid helium by scanning a small sensor. Spatial structures were reported indeed, but only at extremely high-power densities, near the critical heat flow of superfluid helium. ${ }^{13}$
In this paper, we report on a much more sensitive method to resolve the phonon-emission rate spatially. This technique allowed us to investigate larger junctions at much weaker injection rates than previously used. ${ }^{11,12}$ Thus, the local onset of the instability and its growth as a function of the injection current could be observed for the first time. We find that the quasiparticles diffuse towards the regions with higher density and that the injection rates required for the onset of the instability are surprisingly low.

Our samples were traditional $^{3-6}$ triple-film doublejunction structures. The three films consisted of granular A1 of about $1200-\AA$ thickness and had $T_{c}$ 's of about 2.0 $K$. The top and the middle films formed the injector junction to produce the nonequilibrium states. The middle and the bottom films served as detector junction to monitor the gap depression. The area of either junction was $2 \mathrm{~mm} \times 1.7 \mathrm{~mm}$. Geometry prevented tunneling between the top and the bottom film. The overlap of the two junctions was about $90 \%$ of their respective areas.

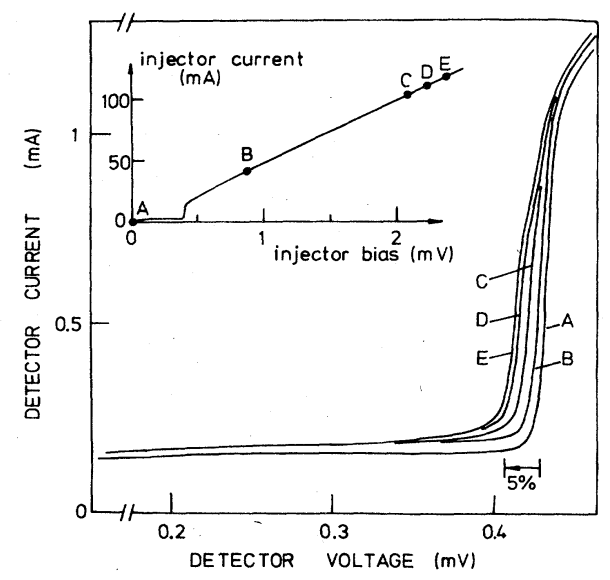

FIG. 1. Current-voltage characteristics of a triple-film double-junction arrangement. Inset: Injector junction with operating points (capital letters) at which the detector characteristics (main figure) were measured. At higher injector biases double-gap structures formed in the detector characteristics. 
The ambient temperature was $1.2 \mathrm{~K}$ and no external magnetic field was applied.

The inset in Fig. 1 shows the $I-V$ characteristic of the injector with those operation points indicated for which the detector characteristics are presented in the main figure. The gap reduction for injector voltages well above $2 \Delta / e$ is clearly visible, but never exceeded $5 \%$ in any of our experiments.

The technique for detecting the spatial distribution of phonon emission is based on the fountain effect of superfluid ${ }^{4} \mathrm{He}$. The junction was held in a horizontal position about $1 \mathrm{~mm}$ above the liquid level, with the sapphire substrate partly immersed, so that a superfluid film of $50-\mathrm{nm}$ thickness formed on top of it. A local rise of the temperature then led to a locally increased helium-film thickness. This technique was first used by Eisenmenger ${ }^{14}$ to resolve phonon-focusing patterns. We have greatly improved the sensitivity by reading out the film thickness interferometrically. For this purpose, a parallel $\mathrm{He}-\mathrm{Ne}$ laser beam incident on the sample was reflected partly from the surface of the tunnel junction and partly from a glass plate $1 \mathrm{~cm}$ above the sample which was slightly tilted. The interference of the two reflected beams resulted in a pattern of parallel fringes as long as the helium film had uniform thickness. Given the refractive index of ${ }^{4} \mathrm{He}$ at $1.2 \mathrm{~K}$ of $n=1.029$, a local increase in film thickness of $10.7 \mu \mathrm{m}$ resulted in a local shift of the interference pattern by one fringe. We could resolve $1 / 20$ fringe or $0.5 \mu \mathrm{m}$, corresponding to a local temperature change of the order of $10^{-5} \mathrm{~K}$ in the helium film. As a check we heated a normal metal film with the size of the junctions. Figure 2 shows photographs of the interference patterns; Fig. 2(a) was taken without power being applied to the film. All fringes are straight lines indicating a constant $\mathrm{He}$-film thickness. At a power level of about $1 \mathrm{~mW} / \mathrm{cm}^{2}$ the fringes started to shift homogeneously over the whole area. In Fig. 2(b) this shift is obvious from the curved lines. The onset occurred within $1 \%$ or $2 \%$ of the applied power which is due to the rapid decrease of the Van der Waals force with distance from the surface. We made use of this sudden onset behavior to emphasize small inhomogeneities in the phonon emission. No hysteretic behavior was observed.

Results for the distribution of the phonon emission in the junction of Fig. 1 are presented in Fig. 3. The un-

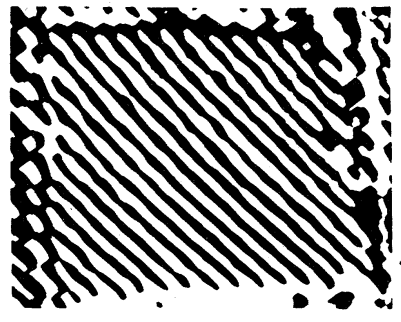

(a)

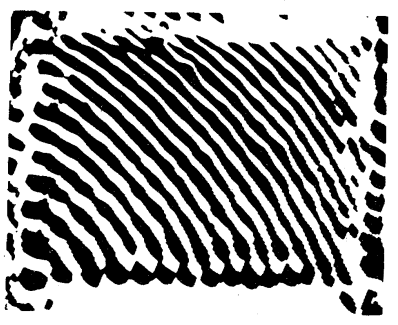

(b)
FIG. 2. Interferograms of a normal metal film. In (a) no power was applied. The straight lines indicate a spatially constant $\mathrm{He}$ film thickness. In (b) a power density of $1.4 \mathrm{~mW} / \mathrm{cm}^{2}$ was dissipated. The fringes shifted over the whole area.
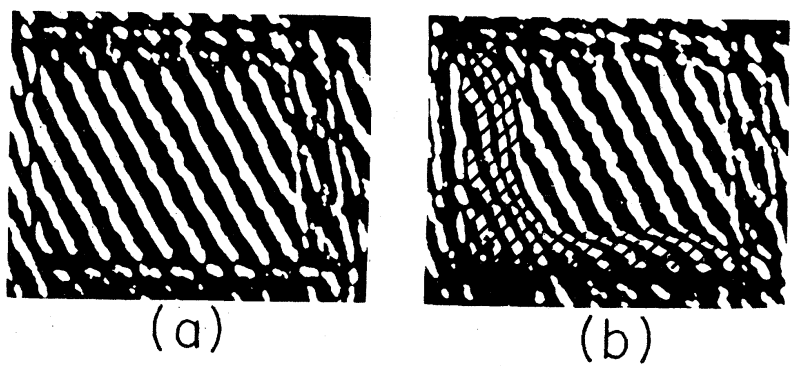

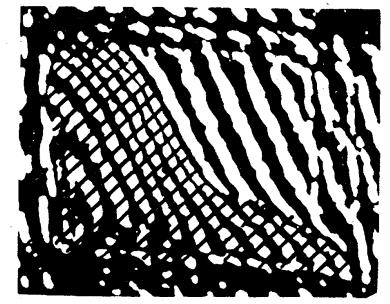

(c)

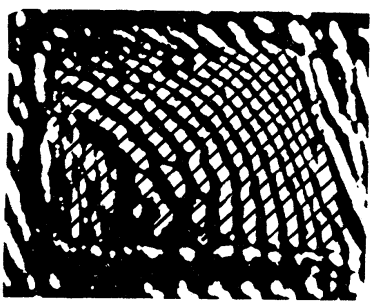

(d)
FIG. 3. Interferograms of the junction of Fig. 1. The hatching indicate the areas where the fringes have been curved. Figures (a), (b), (c), and (d) correspond to operating points $A, C, D$, and $E$ of Fig. 1, respectively. At point $E$ the total dissipated power density was about $8 \mathrm{~mW} / \mathrm{cm}^{2}$. The structures near the border of the photographs are due to the edges of the A1 films which form the tunnel junctions. The injection-current contacts were at the left and at the right side of the photograph.

biased junction, covered with a homogeneous helium film, is shown in Fig. 3(a), for reference. In Fig. 3(b), the injector was biased to operation point $C$. At this point, part of the interference fringes was shifted with respect to the reference pattern indicating clearly that a new phase with higher phonon emission had formed. As a guide to the eye, we have hatched the region where the film became thicker than $3 \mu \mathrm{m}$ or $\frac{1}{4}$ fringe. The first onset of this new phase became already visible at operation point $B$. In Figs. 3(c) and 3(d), the injection was increased to operation points $D$ and $E$, respectively. The new phase grew until it was covering the whole junction. At the same time, a gradual decrease of the second gap was observed as shown in Fig. 1.

In the junction of Fig. 1, the new phase always formed at the same spot, and one may imagine that it was induced by an inhomogeneous tunneling injection. In some junctions, however, the new phase formed at random, an example of which is shown in Fig. 4. In this case, an injection current of $60 \mathrm{~mA}$, corresponding to a voltage of $3.7 \Delta / e$, was repeatedly switched on and off, and the pattern was different every time.

In some other samples it was possible to induce the nucleation at will by focusing a laser spot of $0.1-\mathrm{mm}$ diameter and a power of about $10 \mu \mathrm{W}$ onto the junctions. This is demonstrated in Fig. 5. In Fig. 5(b), only the injector junction was switched on, without using the laser. The injector bias was $\mathrm{eV}=5.0 \Delta$, and the second phase formed as previously. In Fig. 5(c), on the other hand, the laser was on and the injector off. The absorbed laser power led to a comparable phonon emission. Finally, with the laser still 


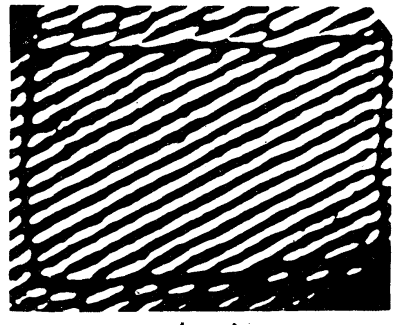

(a)

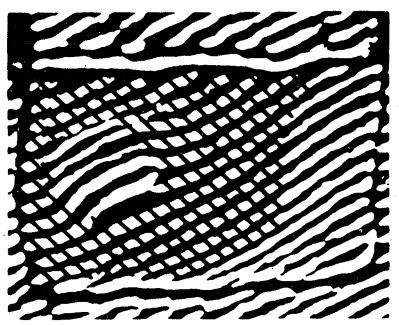

(c)

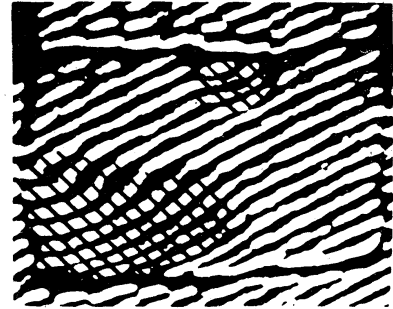

(b)

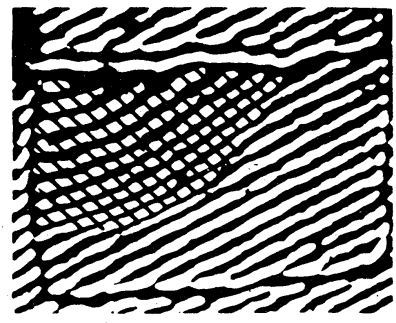

(d)

FIG. 4. Interferograms of a second sample. In (a) no injection. In (b) through (d) the injector bias was set to $3.7 \Delta / e$ each time. This corresponded to a power density of $1.2 \mathrm{~mW} / \mathrm{cm}^{2}$. The instabilities formed in different areas. The current contacts were at the top and the bottom of the photograph.

on, the injector current was turned on again, and only now the laser was switched off. This resulted in Fig. 5(d), with the enhanced phonon emission stable at the new place as long as the current was on.

The fact that we did observe spatial structures supports the previous findings, ${ }^{6,10,11}$ although the actual patterns were different at our low-power densities. ${ }^{15}$ Furthermore, Figs. 4 and 5 give positive proof, for the first time, of the fact that the patterns are intrinsic to the superconductors, and not caused by inhomogeneous tunnel injection. In view of the homogeneous injection, the inhomogeneous emission reveals a lateral diffusion of quasiparticles into the regions of enhanced phonon emission.

At first glance it may be surprising that the size of these regions is sometimes much larger than the quasiparticle diffusion length $(100 \mu \mathrm{m}){ }^{16}$ However, due to the onset behavior of the fringe shifting, the phonon emission (or quasiparticle density) needs to be enhanced by only a few percent to cause an effect. This state can be reached with a diffusion length smaller than the size of the structures.

The regions with enhanced phonon emission are clearly identical with the new phase, since they always grow in size with increasing injection (Fig. 3) and sometimes remain at formerly weakened spots (Fig. 5). Thus we have the fact that the quasiparticles diffuse towards the regions of already enhanced quasiparticle density, a

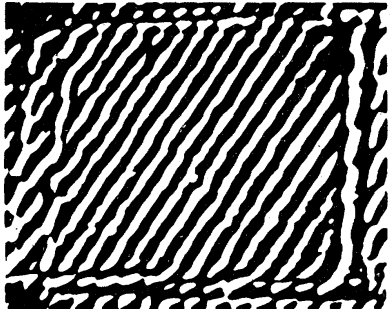

(a)

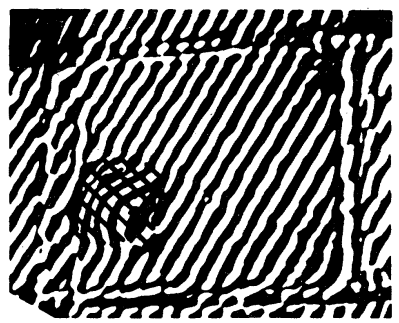

(c)

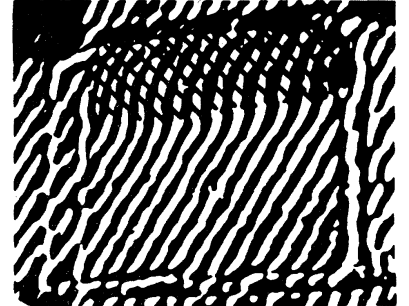

(b)

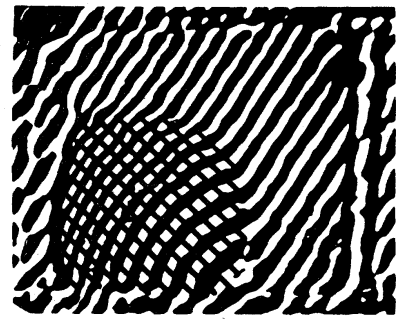

(d)
FIG. 5. Interferograms of a third sample; (a) no injection; (b) injector bias $5.0 \Delta / e$ (power density: $2.2 \mathrm{~mW} / \mathrm{cm}^{2}$ ); (c) no injection but with a laser focused onto the junction; (d) laser off after the bias was again set to $5.0 \Delta / e$. The laser irradiation induced the instability in another area. The injection-current contacts were at the top and at the right side of the photograph in this sample.

behavior which can formally be described by a negative diffusion coefficient. ${ }^{7,8}$

Elesin's theory is therefore not applicable to our experiment in any form, ${ }^{10}$ and one tends to favor the phenomenological models ${ }^{7,8}$ despite their shortcomings. ${ }^{9}$ One should note, however, that these models always require a high-injection threshold for the instability to occur. This implies an appreciably depressed gap in the new phase. In contrast, we have observed the instability at very low injection already, when the gap was depressed by less than $2 \%$. This is well below any conceivable nonlinear regime.

We conclude that the double gaps at high-voltage tunnel injection are now well established experimentally but neither theoretical explanation appears to be satisfying. The "quasiparticle droplets" observed here are somewhat reminiscent of electron-hole droplets in semiconductors. Thus, one way to find a new explanation may be to look more closely into the attractive two-quasiparticle interaction which is inherent in the Fröhlich model of superconductivity. ${ }^{17}$.

Useful discussions with U. Eckern and P. Wölfle and experimental contributions by $\mathrm{W}$. Zoller are gratefully acknowledged.
${ }^{1}$ Nonequilibrium Superconductivity, Phonons, and Kapitza Boundaries, edited by K. E. Gray (Plenum, New York, 1981). ${ }^{2}$ S. M. Faris, S. I. Raider, W. J. Gallagher, and R. E. Drake, IEEE Trans. Magn. 19, 1293 (1983).
${ }^{3}$ C. C. Chi and J. Clarke, Phys. Rev. B 20, 4465 (1979).

${ }^{4}$ R. C. Dynes, V. Narayanamurti, and J. P. Garno, Phys. Rev. Lett., 39, 229 (1977).

${ }^{5}$ K. E. Gray and H. W. Willemsen, J. Low Temp. Phys. 31, 911 
(1978).

${ }^{6}$ I. Iguchi and D. N. Langenberg, Phys. Rev. Lett. 44, 486 (1980).

${ }^{7}$ D. J. Scalapino and B. A. Huberman, Phys. Rev. Lett. 39, 1365 (1977).

${ }^{8}$ I. Iguchi and H. Konno, Phys. Rev. B 28, 4040 (1983).

${ }^{9}$ G. Schön, Physica B 109\&110, 1677 (1982).

${ }^{10}$ V. F. Elesin and Yu. V. Kopaev, Usp. Fiz. Nauk 133, 259 (1981) [Sov. Phys. Usp. 24, 116 (1981)]; U. Eckern, A. Schmid, M. Schmutz, and G. Schön, J. Low Temp. Phys. 36, 643 (1979).

${ }^{11}$ H. Akoh and K. Kajimura, Physica B 107, 537 (1981).

${ }^{12}$ S. Kotani, Y. Suzuki, and I. Iguchi, Phys. Rev. Lett. 49, 391 (1982); I. Iguchi and Y. Suzuki, Phys. Rev. B 28, 4043 (1983).

${ }^{13}$ In Ref. 12, the power density dissipated in the junction was about $4 \mathrm{~W} / \mathrm{cm}^{2}$, close to "non film boiling" of superfluid heli- um; see, e.g., J. A. Clark and R. M. Thorogood, in Cryogenic Fundamentals, edited by G. G. Haselden (Academic, London and New York, 1971), p. 92.

${ }^{14} \mathrm{~W}$. Eisenmenger, in Phonon Scattering in Condensed Matter, edited by H. J. Maris (Plenum, New York and London, 1980), p. 303.

${ }^{15}$ Patterns which were similar to ours have very recently been observed by Y. Nisida, T. Nishimura, A. Nishiura, and I. Iguchi, Solid State Commun. 50, 553 (1984). They used Sn which may have led to the shorter length scale of their patterns.

${ }^{16}$ S. Y. Hsieh and J. L. Levine, Phys. Rev. Lett. 20, 1502 (1968).

${ }^{17}$ N. N. Bogoliubov, V. V. Tolmachev, and D. V. Shirkov, $A$ New Method in the Theory of Superconductivity (Consultants Bureau, New York, 1959), Chap. 4. 